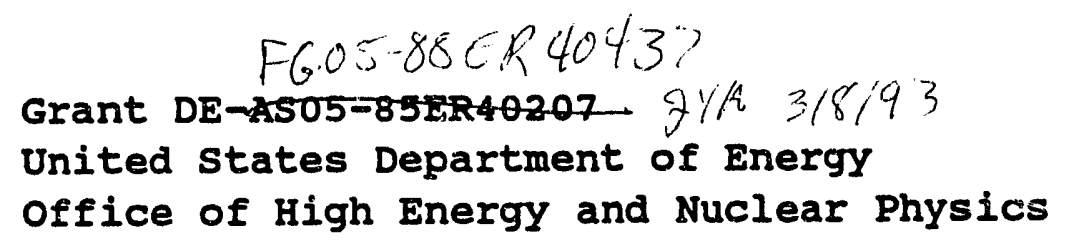

PROGRESS IN RESEARCH

1992

PION CORRELATIONS AND CALORIMETER DESIGN

FOR HIGH ENERGY HEAVY ION COLLISIONS

\author{
Kevin L. Wolf \\ Cyclotron Institute \\ Texas A\&M University \\ College station, Texas 77843
}

\title{
DISCLAIMER
}

account of work sponsored by an agency of the United States This report was prepared as an account hovernment nor any agency thereof, nor any of their Government. Neither the United States Government nor assumes any legal liability or responsiemployees, makes any warranty, express or implied, or assu information, apparatus, product, or bility for the accuracy, completeness, or usefulness of any informate privately owned rights. Referprocess disclosed, or represents that its use would not infinge privale by trade name, trademark, ence herein to any specific commercial product, process, or servimply its endorsement, recommanufacturer, or otherwise does not necessarily constute or any agency thereof. The views mendation, or favoring by the United States Government or anstate or reflect those of the

and opinions of authors expressed herein do not 
PION CORRELATIONS AND CALORIMETER DESIGN FOR HIGH ENERGY HEAVY ION COLLISIONS

TABLE OF CONTENTS

INTRODUCTION

page 1

I. THE EOS TIME PROJECTION CHAMBER PROGRAM 1

II. EXPERIMENT NA44 AT CERN 4

III. SAMPLING CALORIMETER CONSTRUCTION 5

IV. DATA ANALYSIS FOR NA44 8

v. RECENT PUBLICATIONS 11

\section{RESEARCH STAFF}

K.L. Wolf, Professor

A.D. Chacon, Research Associate

M. Murray, Research Associate

R.K. Choudhury, visiting scientist

H. Jabs, Graduate Student

s. Potter, Computer System Manager

B. Sadhwani, ACAD Design

S. Johnson, Calorimeter R\&D

M. Lafrance, Calorimeter R\&D

D. Williams, Calorimeter R\&D 


\section{INTRODUCTION}

The schedule calling for the permanent shutdown of Bevalac operations early in calendar year 1993 put a great deal of pressure on the EOS time projection chamber group during the past year. A highly concentrated effort was necessary for the completion of the physics program on this new detector system, essentially in one running period and with the lack of feedback from data analysis. of course the rather mature area of heavy-ion collisions at Bevalac energies from past studies required that high statistics complete data be taken. To this end physics was emphasized that used newly developed beams such as ${ }^{58} \mathrm{Ni}$ at the highest Bevalac energies and the heaviest beams such as ${ }^{197} \mathrm{Au}$, studies of which in the past had suffered from design limitations in the detectors. Lower statistics data sets were taken on many other systems, especially in order to match up with and to extend past studies done with other types of decectors. A large analysis effort will now follow, with the principal emphasis from TAMU on identical pion interferometry. With the end of the Bevalac operation, it was necessary to plan the physics program for the next few years based at a different facility. It was decided to pursue identical particle interferometry and the measurement of identified single particle spectra with the NA44 collaboration at CERN. While the physics goals of the NA44 collaboration coincided with our interests, also the timeliness of the project in terms of development for the lead beam upgrade at the SPS in 1994, and the increased beam energy relative to our past experiments at the Bevalac and at the AGs were important factors. An electromagnetic sampling calorimeter test section that features a highly developed optical fiber readout has been constructed in a continuation of our calorimeter development started in 1987. The 100-tower structure was constructed as a cost effective device optimized in segmentation and energy resolution for a high multiplicity environment expected at RHIC or the LHC.

\section{The EOS Time Projection Chamber Project}

Relativistic heavy-ion research at the Bevalac opened a major new field of nuclear science in the study of the dynamics of nucleusnucleus collisions where baryon and meson degrees of freedon are excited. Experiments with large acceptance devices such as the 
Streamer Chamber Facility and the Plastic Ball produced results that were suggestive of a dependerice on parameters of the nuclear matter equation of state. However, pion multiplicity measurements that were linked to the stored compressional energy through intranuclear cascade model calculations have not stood up to more detailed calculations $1,2,3$. Although no calculation can consistently account for pion multiplicities in nucleon - and heavy-ion-induced reactions as a function of beam energy and centrality, the discrepancies are attributed to the complexities of pion absorption rather than the stored compressional energy. Similarly the emission patterns of single pion inclusive data in the $1 \mathrm{~A} \mathrm{GeV}$ beam energy regime have not been explained4. Presently models that fit event shapes from emitted protons and clusters have come into question with regard to the sensitivity to the nuclear matter equation of state. Previously it was been known that medium effects and other factors can greatly complicate the extraction of an incompressibility constant from the event shapes for data in the Bevalac energy range. As outlined in last year's proposal, an interpretation of results from a model based on quantum molecular dynamics 5 suggests that the stored compressional energy is extremely small, only a few $\mathrm{MeV} /$ nucleon for heavy-ion collsions in the 0.4-0.8 A GeV energy range where experimental "flow effects" are large. The flow effects are considered to be the result of nucleon-nucleon forces in the entrance channel instead of a response from stored compressional energy. The QMD calculations agree qualitatively with earlier calculations based on the BUU model; but the earlier calculations were not analyzed specifically in terms of stored compressional energy. Whether or not the current conclusions from QMD calculations are correct, higher quality experimental data over a wide range of systems are needed to test the current models. We do consider the current QMD calculations to have some validity and consider the highest Bevalac energies to be the most important in equation of state studies.

Most energy flow analyses eliminate pions from consideration because of the high emission velocities relative the velocities of heavier particles and relative to expected flow velocities. Futhermore extra dispersion is introduced due to delta decay kinematics. A goal of this program is to investigate pion emission patterns and use pion interferometry from the EOS experiments to measure sizes and shapes of the interaction zone. The measurement of particleexclusive data to determine the reaction plane and the flow parameters provides a powerful method to characterize the event class. Although pions provide information at a rather late stage of the reaction, a comparision with flow analyses should prove to be 
quite interesting with regard to the validity of assumptions about compression.

The feasibility of performing two pion correlations in the EOS TPC was reported in last year's progress report and found to be quite promising in terms of two-track resolution and efficiency. The major problem with a high acceptance device like the present TPC is in obtaining sufficient numbers of events i.e. close correlated pion pairs. Optimized small acceptance angle multiparticle spectrometers usually have a considerable advantage in that regard, and several sets of such high statistics data have been taken for heavy-ion reactions at LBL, BNL and CERN. From an initial scan of the data taken in the completed EOS program, at least two sets of data are believed to have sufficient statistics to provide good three dimensional pion source parameters, each of more than 50,000 close correlated pairs. Both cases represent the highest energies available at the Bevalac in the respective mass ranges, namely $1.9 \mathrm{~A} \mathrm{GeV} 58 \mathrm{Ni}+\mathrm{Cu}$ and $1.2 \mathrm{~A} \mathrm{GeV} \mathrm{Au}+\mathrm{Au}$. The analysis effort for pion correlations will be focused on these high statistics data sets with comparisions made later to the complete event parameters.

In preparation for the past year's Bevalac experiments, considerable care had to be given to the TPC and to the external detectors forming the system shown in Fig.1, especially since there would be no second round of follow up experiments. To this end one of the weak links in the external detectors was in the time-of-flight wall which provides mass and charge identification for fragments at small angles relative to the beam axis. Several years ago the reponse of this detector mysteriously decreased to essentially eliminate the usefulness for light fragments with $\mathrm{z}=1-3$. The 98 slats of $1.7 \mathrm{~m}$ plastic scintillator were made of the same material as covered in our progress report last year on calorimetry where we reported a factory-wide instability of Bicron plastic scintillator for runs the past four years. A combination of poor scintillator and subsequent poor procedure in the bending and handling done at LBL had resulted in severe crazing and poor light transmission down the rather narrow thin scintillator slats to PMTs on either end. Tests in our laboratory indicated attenuation factors ranging from 3 to 10 relative to high quality scintillator, which in turn made the photon statistics too low for detection of low $\mathrm{z}$ particles. In cooperation with Bicron corporation for a accelerated production schedule, scintillator was bent and wrapped in our TAMU scintillator labs and installed in collaboration with the scharenberg group from Purdue to bring the response of the detector up to a useful level. Mip response $(\mathrm{z}=1)$ was attained reliably in the data. 
The importance of obtaining mip response impacts two types of measurements. The projectile fragmentation studies are attempting to perform particle exclusive measurements, but as we learned several years ago, the major portion of the mass in the projectile cone is carried by light particles. Secondly, the central collision studies benefit from $p, d, t$ identification in the small angle near-projectile frame, and cannot be identified well with the TPC ionization measurements alone.

\section{EXPERIMENT NA44 AT CERN}

Collaborating Institutions: BNL, CERN, Columbia, Creighton, Hiroshima U., LANL, Lund, Tbiliisi state U., Tsukuba National Lab., Neils Bohr Inst., Ohio State, Tokyo U., and Tsukuba U.

Fixed target operation at a beam energy or $200 \mathrm{~A} \mathrm{GeV}$ is considered to be the highest energy that is practical before going to collider mode as planned at RHIC. Essentially, the energy regime is the same as that investigated for $p+p$ and ${ }^{4} \mathrm{He}+{ }^{4} \mathrm{He}$ at the ISR collider. While well below the energy necessary for transparency and the formation of a baryon free high energy density region for the quark gluon plasma, the energy density may be sufficient for the formation of a mixed-phase QGP. But more realistically, it is important to map out and understand the features of heavy-ion reactions both at BNL and CERN, at energies lower than the future colliders in order to be able to understand those later data.

Identical particle interferometry can provide information about the dynamical evolution of heavy-ion reactions, specifically through the space-time extent of the emitting source. The time extent of particle emission can provide evidence for a phase transition as outlined by Bertsch and Brown ${ }^{7}$. The relatively high energy available at CERN allows interferometry measurements with several types of produced particles including pions, kaons and possibly antiprotons in the lead beam experiments, and provide corresponding source sizes and shapes. An additional factor at CERN energies, however is the large amount of resonance production and deexcitation that can confuse or dilute interferometry measurements. The resonance decay contribution is considered to be an important area of study in these experiments. High quality identified single particle spectra are a product of these expeiments which are necessary and quite valuable in understanding the topology of the reactions. The shapes of the spectra and the particle ratios are a constant topic of discussion in the 
understanding of the dynamics of the reactions and even in the search for exotic effects.

NA44 is an example of a second generation experiment, which builds upon the results of the completed and analyzed survey experiments. The first phase of the apparatus was brought on line for recent $200 \mathrm{~A} \mathrm{GeV}$ sulfur beam periods at the SPS and for $450 \mathrm{GeV}$ proton beams. The general layout of the apparatus was designed for very heavy ion beams originally as shown in Fig.2, but not completely instrumented at that time. Because of the increased multiplicity with lead beams and because of experience gained in the previous program, some new equipment must be built for the lead beam SPS capability due in 1994. The general philosopy is one of attempting to measure a small slice of an event instead of a global measurement, and to make that measurement in an optimized manner. Thus the primary goal is to measure identified single paricle distributions and two particle distributions at or near midrapidity. The primary detector is a focusing spectrometer designed to accept multiple particles with small momentum difference and uses a combination of three quadrupoles, three dipole magnets, and a series of TOF hodoscopes, MWPCs and Cherenkov counters to track and identify charged particles in the spectrometer. Thus pions, kaons and protons both positively and negatively charged are identified and measured with good electron rejection. A typical data sample for pion pairs consists of 200,000 events. The primary trigger is furnished by a plastic scintillator that operates in a positive pulse height mode, i.e. as compared to a leading particle veto trigger. A silicon pad array is used off line to determine the charged-particle multiplicity over the rapidity range of $1.5-$ 3.3. Progress on the analysis of data collected is given in section IV.

\section{SAMPLING CALORIMETER DEVELOPMENT AND TESTS}

An optical fiber readout srheme for sampling calorimetry was developed through the first stages at TAMU in 1987 before starting The Participant Calorimeter ${ }^{8}$ project in collaboration with LANL. The original scheme outlined by Albrow, et al ${ }^{9}$ was extended to provide much higher, more reproducible response from plastic scintillator plates, into each of which was bonded a single $1.5 \mathrm{~mm}$ diameter clad plastic optical fiber. The index of refraction of the bonding agent was matched to the fiber cladding. The green fiber used had the property of light absorption in the UV and blue, and reemission in the green after fairly efficient shifting. The light 
is then trapped and transmitted efficiently, with a transmission length of typically 15-20 meters. Most of the development time was spent on handling, grading and bonding fibers with little in the way for optimization of the method, since the photon response obtained was quite sufficient for a hadronic calorimeter. After some more recent development time on a DOE Generic Detector project it is found that the response can be made as high as 20-30 photoelectrons per plate for mips through a $3 \mathrm{~mm}$ thick plastic scintillator and 5 pe response for $1 \mathrm{~mm}$ thick scintillator.

For calorimetry, the superior response allows compensated hadronic calorimeters to use much thinner lead and scintillator plates, and thus allows an increase in the energy resolution to that of uranium calorimeters, and allows compact high resolution electromagnetic calorimeters to be constructed, through the good photoelectron statistics and uniform response. The disadvantages of the method include the labor intensiveness in bonding and stacking, and the dead regions caused by the fiber exit channels when one fiber per plate (or a few plates) is used. Also a large fiber bundle requires a large area readout device which is usually a phototube and always is expensive.

A solution to the readout problem was developed whereby the light from an entire calorimeter tower is concentrated into a single small optical fiber. Wavelength shifting fibers run perpendicular to the scintillator plane in a tile-type structure and a single $1.5 \mathrm{~mm}$ fiber reads out 75 plates as shown in Fig.3. In terms of an integrated solution the current scheme essentially eliminates dead regions, and allows the use of small active readout devices such as segmented anode phototubes as used here. The cost of the active readout device is then reduced by one order of magnitude per channel compared to conventional PMTs, most significant in an EM calorimeter. In the development project for a 100-tower test section constructed in our laboratory, a response of 5 photoelectrons per plate was obtained from $3 \mathrm{~mm}$ thick scintillator and a single $1.5 \mathrm{~mm}$ optical fiber readout. In the actual test section provision was made for up to 4-fold longitudinal segmentation which allows good particle identification over the 25 radiation lengths. An entire tower is read out by a single fiber, but additional light is collected from preshower and shower peak sections using aluminized fiber where light collection is unwanted. The energy resolution is determined by the $2 \mathrm{~mm}$ thick lead absorber plates at approximately $78 /$ SQRT(E). Problems with uniformity of response in a similar early scheme 10 have been solved by developing a "highly reflective" solution for the light collection as covered 
in last year's progress report. Optimized scintillator, reflector and optical fiber combinations have reduced nonuniformities to the few percent level in bench tests. The good performance and the economy due to a small area readout device allows a high granularity necessary for measurement of electromagnetic energy in a high multiplicity such as at RHIC or the IHC.

The STAR collaboration has made provisions for an older type of fiber optic calo:imeter with 1 fiber per 1-2 plates and extremely large towers following development of lead casting techniques at ANL. Although called an EM calorimeter, this is a true EM device only for $p+p$ reactions. For heavy-ion reactions such as the $A u+$ Au syatem that controls other design criteria, there is a 300-400\% tower occupancy and more than half of the deposited energy is from charged pions, which cannot be separated or subtracted with the TPC. A shower maximum detector only tells the obvious that the data result from pileup. The principal emphasis is on high energy physics at rather low multiplicities, using polarized beams in socalled spin physics.

\section{REFERENCES}

1. R. Stock, et al Phys. Rev. Lett. 491239 (1982).

2. G. Bertsch, Phys. Rev. C29 673(1984).

3. J. Aichelin, Phys. Rep.202 233(1991).

4. K.L. Wolf, et al, Phys. Rev. Lett. 431449 (1979) •

5. J. Janicke and J. Aichelin, Heidelberg Preprint HD-TVP-91-3 and Nucl. Phys. 547542 (1992).

6. A.D. Chacon, et al, Phys. Rev. C43 2670(1991).

7. G. Bertsch and G.E. Brown, Phys. Rev. C40 1830(1989).

8. J. Simo-Gillo, et al, NIM $\$ 525474(1992)$ and NIM A317 474 (1992).

9. M. Albrow, Nucl. Phys. 4461 417c(1987).

10. H. Fessler, et al, NIM 284 (1985). 


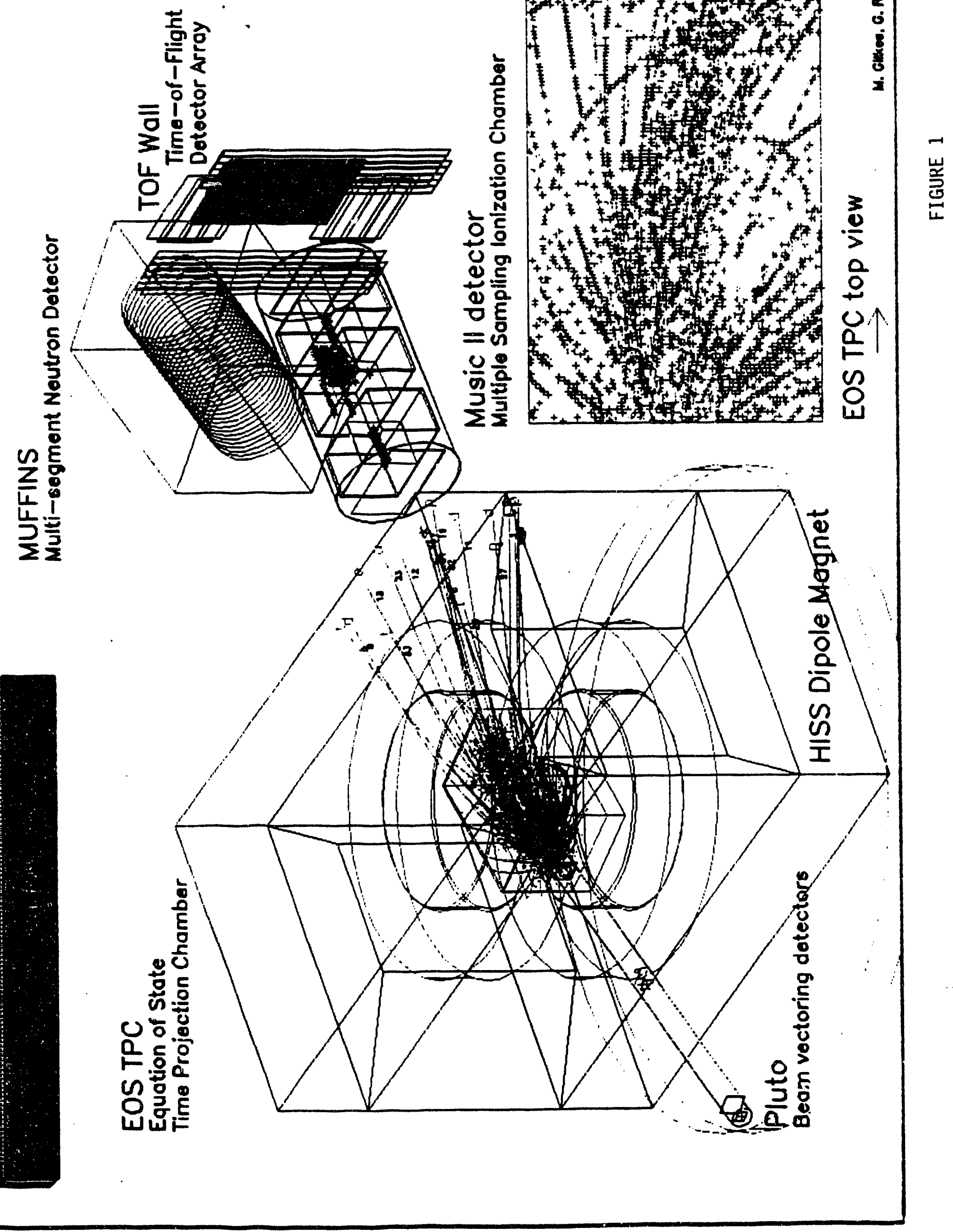




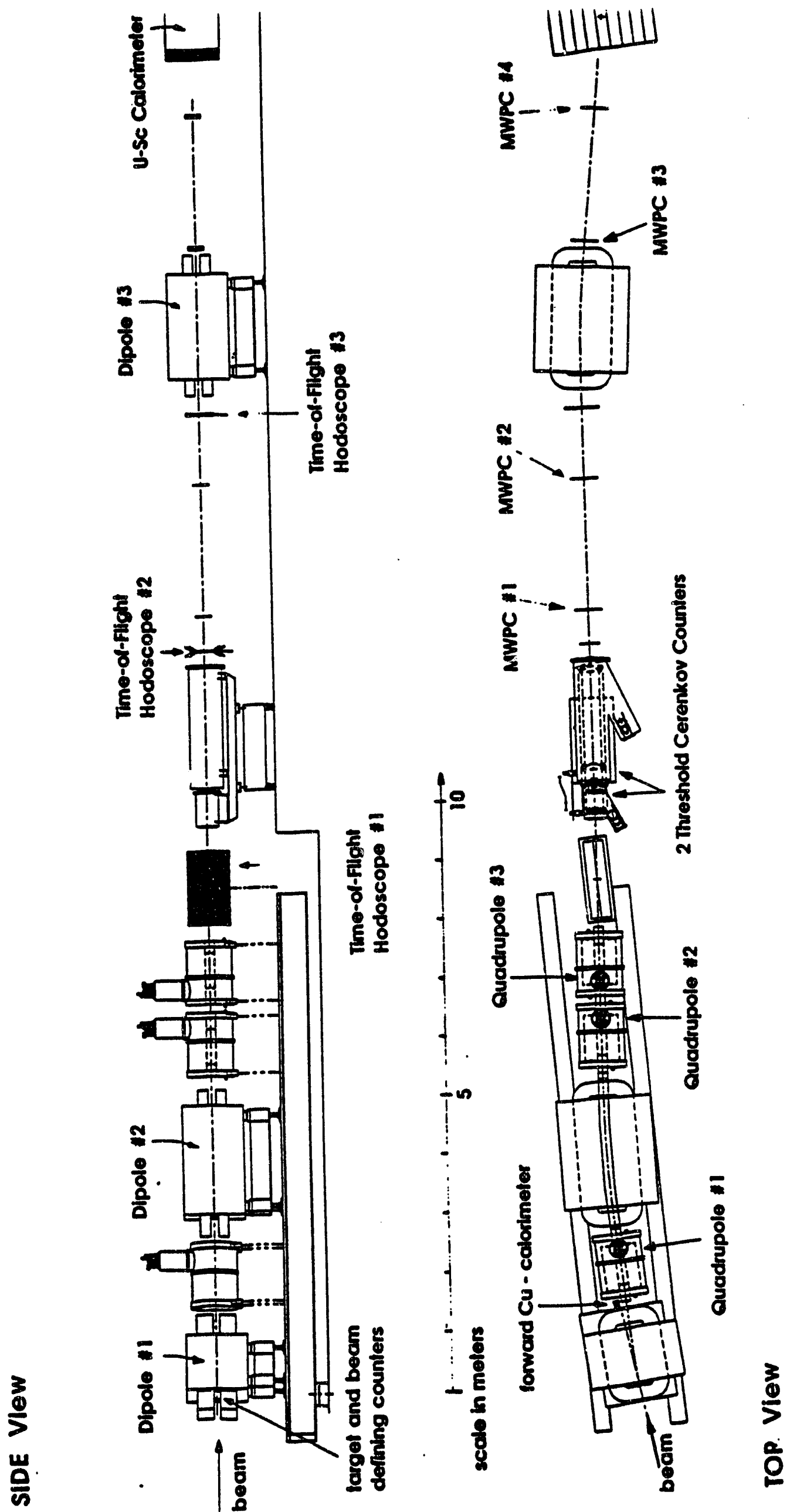




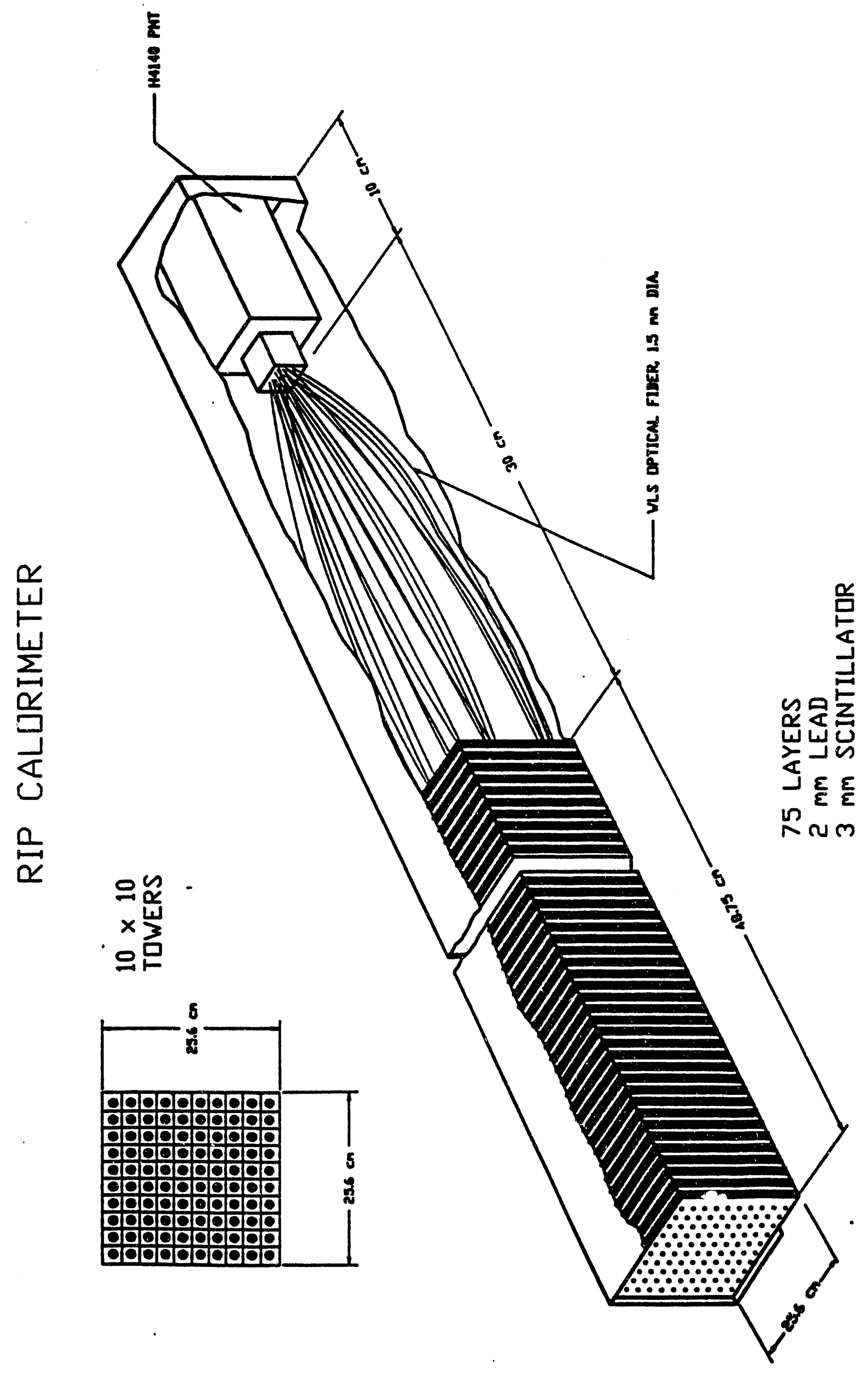




\section{Recent Publications: $1990-92 \quad$ K.I. Wolf}

Midrapidity Pion Ratios in $1.05 \mathrm{GeV} /$ nucleon $4{ }^{\circ} \mathrm{Ca}+{ }^{40} \mathrm{Ca}$ Collisions, J.W. Harris, J. Miller, H.G. Pugh, P. Renteln, G. Roche, P.N. Kirk, G. Krebs, R. Brockmann and K.I. Wolf, Phys. Rev. C41 147 (1990).

Electromagnetic Dissociation of ${ }^{28} \mathrm{si}$ at $\mathrm{E}_{1 \mathrm{ab}} / \mathrm{A}=14.6 \mathrm{GeV} / \mathrm{A}$ by Nucleon mission, J. Barrette, P. Braun-Munzinger, W.E. Cleland, G. David,. E. Duek, M. Fatyga, D. Fox, S.V, Green, J.R. Hall, R. Heifetz, T.K. Hemick, N. Hermann, R.W. Hogue, G. Ingold, K. Jayanada, D. Kraus, A. Legault, D. Lissauer, W.J. Llope, T. Iudlam, R.D. Majka, D. Mackowiecki, S.K. Mark, J.T. Mitchell, M. Muthuswamy, E. o'Brien, L.H. Olsen, V. Polychronakos, M. Rawool-sullivan, F.S. Rontondo, J. Sandweiss, B. Shivakumar, J. Simon, U. Sonnarara, J. Stachel, J. Sunier, H. Takai, T.G. Throwe, H. VanHecke, L. Waters, W.J. Willis, K. Wolf, D. Wolfe and C. Woody, Phys. Rev.c41 1512 (1990).

Energy Flow and stopping in Relativistic Heavy-Ion Collisions at $\mathrm{E}_{\text {lab }} / \mathrm{A}=14.6 \mathrm{GeV}, \mathrm{J}$. Barrette, R. Bellwied, P. BraunMunzinger, w.E. Cleland, G. David, E. Duek, M. Fatyga, D. Fox, A. Gavron, S.V. Green, J. Hall, R. Heifetz, T.K. Hemmick, M. Herman, N. Herrmann, R.W. Hogue, G. Ingold, K. Jayanada, D. Kraus, A. Legault, D. Lissauer, W.J. Llope, T. Ludlam, R.D. Majka, D. Mackowiecki, S.K. Mark, J.T. Mitchell, M. Muthuswamy, E. O'Brien, I.H. Olsen, V. Polychronakos, M. Rawool-sullivan, F.S. Rontondo, J. sandweiss, B. Shivakumar, J. Simon, U. Sonnarara, J. stachel, J. Sunier, H. Takai, T.G. Throwe, H. VanHecke, I. Waters, W.J. Willis, K. Wolf, D. Wolfe and C.I. Woody, Phys. Rev.C41 1512 (1990).

Energy Flow and stopping in Relativistic Heavy-Ion Collisions at $E_{l a b} / A=14.6 \mathrm{GeV}, J$. Barrette, R. Bellwied, P. Braun- 
Muruinger, W.E. Cleland, G. David, E. Duek, M. Fatyga, D. Fux, A. Gavron, S.V. Green, J. Hall, R. Heifetz, T.K. Hemmick, M. Herman, N. Herrmann, R.W. Hogue, G. Ingold, K. Jayanada, D. Kraus, A. Legault, D. Lissauer, W.J. Llope, T. Ludlam, R.D. Majka, D. Mackowiecki, S.K. Mark, J.T. Mitchell, M. Muthuswamy, E. O'Brien, I.H. Olsen, V. Polychronakos, M. Rawool-sullivan, F.S. Rontondo, J. Sandweiss, B. Shivakumar, J. simon, U. Sonnarara, J. stachel, J. Sunier, H. Takai, T.G. Throwe, H. VanHecke, L. Waters, W.J. Willis, K. Wolf, D. Wolfe and C.I. Woody, Phys. Rev. Lett. $\underline{64} 1219$ (1990).

Search for strange Quark Matter in High-Energy Heavy-ion Collisions, J. Barrette, R. Bellwied, P. Braun-Munzinger, W.E. Cleland, G. David, E. Duek, M. Fatyga, D. Fox, S.V. Green, J.R. Hall, R. Heifetz, T.K. Hemmick, N. Herrmann, R.W. Hogue, G. Ingold, K. Jayanada, D. Kraus, A. Legault, D. Lissauer, W.J. Llope; T. Ludlam, R.D. Majka, D. Mackowiecki, S.K. Mark, J.T. Mitchell, M. Muthuswamy, E. o'Brien, L. Olsen, V. Polychronakos, M. Rawool-Sullivan, F.S. Rontondo, J. Sandweiss, B. Shivakumar, J. Simon, A.J. slaughter, U. Sonnarara, J. Stachel, J. Sullivan, J. Sunier, H. Takai, T. Throwe, H. Van Hecke, I. Waters, K.L. Wolf, D. Wolfe, and C. Woody, Phys. Lett. B252 530(1990).

Response of a Sampling Calorimeter to Low Energy Particles, M.W. Rawool-Sullivan, J. Shoemaker, J. Simon, J.P. Sullivan, K.I. Wolf, A. Archuleta, G. Barasch, P. Bennett, D. Bertini, J. Bossevain, K. Holtzscheiter, B. Jacak, W. Sondheim, J. Sunier, H. Van Hecke and B. Wolf, Nucl. Phys. A525 677c(1991).

Pion Correlations in Relativistic Heavy Ion Collisions for Three symmetric systems, A.D. Chacon, J.A. Bistirlich, R.R. Bossingham, H. Bossey, H.R. Bowman, C.W. Clawson, K.M. Crowe, T.J. Humanic, M. Justice, P. Kammel, J.M. Kurck, S. Ijungfelt, C.A. Meyer, C. Petijean, J.O. Rasmussen, M.A. stoyer, O. Hashimoto, Wm. C. Mcharris, J.P. Sullivan, K.I. Wolf and W.A. Zjac, Phys. Rev. C43 2670 (1991). 
The Design and Construction of a Lead/scintillator sampling Calorimeter with Fiber optic Readout, J.simon-Gillo, A. Faroog, M.W. Rawool-sullivan, A. Ray, J. Shoemaker, J.P. Sullivan, K.I. Wolf, E.F. Barasch, J.G. Bossevain, D. Fox, A. Gavron, K. Holzscheiter, B.V. Jacak, T. Lopez, W. Sondheim, J.W. Sunier, H. Van Hecke and B. Wolf, NIM 525 677 (1991).

Forward Baryons in Relativistic Nucleus-Nucleus Collisions, J Barrette, R. Bellwied, P. Braun-Munzinger, W.E. Cleland, G. David, J. Dee, M. Fatyga, D. Fox, S.V. Green, J. Hall, T.K. Hemmick, R. Heifetz, N. Herrmann, R.W. Hogue, G. Ingold, K. Jayanada, D. Kraus, B. Shiva Kumar, M. Lisa, D. Lissauer, W.J. Llope, T. Ludlam, R. Majka, D. Mackowiecki, S.X. Mark, J.T. Mitchell, M. Muthuswamy, E. O’Brien, V. Polychronakos, C. Puruneau, F. Rontondo, J. Sandweiss, J. Simon, U. Sonnarara, J, Stachel, H. Takai, T. Throwe, I. Waters, C. Winter, C. Woody, K. Wolf, D. Wolfe, and $Y$. Zhang, Phys. Rev C45 819 (1992).

Response of the Participant Calorimeter to $1.5-6.8 \mathrm{GeV} / \mathrm{C}$ Electrons and Hadrons, D.Fox, J. Simon-Gillo, M.W. RawoolSullivan, J.G. Boissevain, W.E. Cleland, A. Gavron, B.V. Jacak, D. Kraus, W. Sondheim, J.P. Sullivan, H. van Hecke, S. Watson, K.I. Wolf, and Z. Zhang, NIM 317 474(1992).

Electromagnetic Dissociation, of Relativistic ${ }^{28} \mathrm{Si}$ into $\mathrm{p}+{ }^{27} \mathrm{Al}$, J. Barrette, R. Bellwied, P. Braun-Munzinger, W.E. Cleland, G. David, J. Dee, O. Dietzsch, E. Deuk, M. Fatyga, D. Fox, S.V. Green, J.R. Hall, T.K. Hemmick, N. Hejrmann, R.W. Hogue, B. Hong, K. Jayanada, D. Kraus, B. Shiva Kumar, R. Lacasse, D. Lissauer, W.J. Llope, T. Ludlam, R. Majka, D. Mackowiecki, S.K. Mark, s. McCorkle, J.T. Mitchell, M. Muthuswamy, E. O'Brien, V. Polychronakos, C. Puruneau, F.S. Rontondo, J. Sandweiss, J. simon-Gillo, U. Sonnarara, J. Stachel, H. Takai, E.M. Takagui, T.G. Throwe, L. Waters, W.J. Willis, C. Winter, K. Wolf, D. Wolfe, C.I. Woody, N. Xu, Y. Zhang, Z. Zhang, and Z. Zou, Phys. Rev. C45 2427 (1992). 
Charged Particle Multiplicity in ${ }^{28} \mathrm{Si}+\mathrm{Al}, \mathrm{Cu}$, and $\mathrm{Pb}$ Reactions at $\mathrm{E}_{\text {lab }}=14.6 \mathrm{GeV} /$ nucleon, J. Barrette, R. Bellwied, P. Braun-Munzinger, w.E. Cleland, G. David, O. Dietzsch, E. Deuk, M. Fatyga, D. Fox, S.V. Green, J.R. Hall, R. Heifetz, T.K. Hemmick, M. Herman, N. Herrmann, R.W. Hogue, G. Ingold, K. Jayanada, D. Kraus, B. Shiva Kumar, R. Lacasse, D. Lissauer, W.J. Llope, T. Ludlam, R. Majka, D. Mackowiecki, S.K. Mark, S. McCorkle, J.T. Mitchell, M. Muthuswamy, E. O'Brien, L. Olsen, V. Polychronakos, C. Puruneau, M. Rawool-sullivan, F.S. Rontondo, J. Sandweiss, J. Simon-Gillo, U. Sonnarara, J. Stachel, J.P. Sullivan, J. Sunier, H. Takai, E.M. Takagui, T. Throwe, H. van Hecke, I. Waters, K. Wolf, D. Wolfe, C.L. Woody, N. Xu, and $z$. zhang, Phys. Rev. C46 312 (1992).

Recent Results from Experiment E814 at Brookhaven, $J$. Barrette, R. Bellwied, P. Braun-Munzinger, W.E. Cleland, T.M. Cromier, G. David, J. Dee, G.E. Diebold, O. Dietzsch, M. Fatyga, D. Fox, J.V. Germani, M. Ghalambor-Dezfuli, $S$. Gilbert, S.V. Green, J.R. Hall, T.K. Hemmick, N. Herrmann, B. Hong, G. Ingold, K. Jayanada, D. Kraus, B. Shiva Kumar, R. Lacasse, D. Lissauer, W.J. Llope, T. Ludlam, R. Majka, D. Mackowiecki, S.K. Mark, S. McCorkle, J.T. Mitchell, M. Muthuswamy, E. O'Brien, L. Olsen, V. Polychronakos, C. Puruneau, F.s. Rontondo, J. Sandweiss, N.C. desilva, J. Simon-Gillo, U. Sonnarara, J. Stachel, H. Takai, E.M. Takagui, T. G. Throwe, C. Winter, G. Wang, I. Waters, $K$. Wolf, D. Wolfe, C.L. Woody, N. Xu, Y. Zhang, Z. Zhang, and c. Zou, Nucl Phys 1544 137(1992).

Momentum Distributions of Light Mass Fragments in si-Nucleus Collisions at $14.6 \mathrm{GeV} /$ Nucleon, J. Barrette, R. Bellwied, P. Braun-Munzinger, W.E. Cleland, T. Cromier, G. David, J. Dee, G.E. Diebold, O. Dietzsch, E. Duek, M. Fatyga, D. Fox, S.V. Green, J.R. Hall, T.K. Hemmick, N. Hermann, B. Hong, G. Ingold, K. Jayanada, D. Kraus, B. Shiva Kumar, R. Lacasse, D. Lissauer, W.J. Llope, T. Ludlam, R. Majka, D. Mackowiecki, S.K. Mark, S. McCorkle, J.T. Mitchell, M. 
Muthuswamy, E. O'Brien, V. Polychronakos, C. Puruneau, F.S. Rontondo, J. Sandweiss, N.C. da silva, J. simon-Gillo, J. slaughter, U. Sonnarara, J. Stachel, H. Takai, E.M. Takagui, T. G. Throwe, C. Winter, L. Waters, K. Wolf, D. Wolfe, C.I. Woody, N. Xu, Y. Zhang, Z. Zhang, and C. Zou, Nucl Phys A544 423 (1992).

Antiproton Production in ${ }^{28}$ si-Nucleus Interactions, $J$. Barrette, R. Bellwied, P. Braun-Munzinger, W.E. Cleland, T. Cromier, G. David, J. Dee, G.E. Diebold, O. Dietzsch, E. Duek, M. Fatyga, D. Fox, S.V. Green, J.R. Hall, T.K. Hemmick, N. Herrmann, B. Hong, G. Ingold, K. Jayanada, D. Kraus, B. Shiva Kumar, R. Lacasse, D. Lissauer, W.J. Llope, T. Ludlam, R. Majka, D. Mackowiecki, S.K. Mark, S. McCorkle, J.T. Mitchell, M. Muthuswamy, E. O'Brien, V. Polychronakos, c. Puruneau, F.s. Rontondo, J. Sandweiss, N.C. da silva, J. simon-Gillo, J. Slaughter, U. Sonnarara, J. Stachel, H. Takai, E.M. Takagui, T. G. Throwe, C. winter, L. Waters, K. Wolf, D. Wolfe, C.I. Woody, N. Xu, Y. Zhang, Z. Zhang, and C. Zou, Nucl Phys 1544 599(1992). 

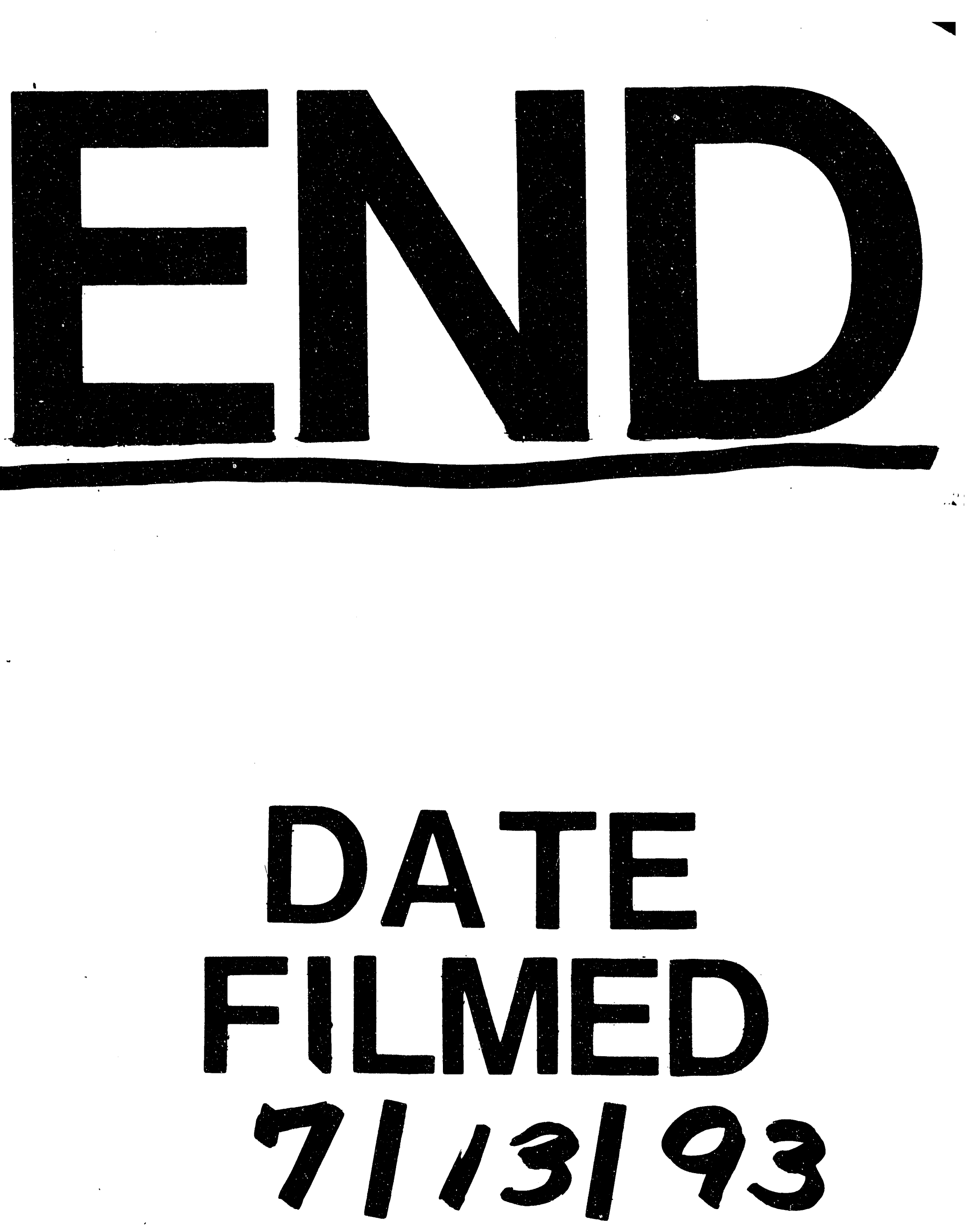
